\title{
Molecular beam epitaxial GaAs optical detectors on silica ilbers
}

\author{
Wei-Oi Li, Albert Chin, and Pallab Bhattacharya \\ Solid-State Electronics Laboratory and Center for High Frequency Microlectronics, Department of \\ Electrical Engineering and Compurer Science, The University of Michigan, Ann Arbor, \\ Michigan $48109-2122$ \\ S. Divita \\ U.S. Army Communications Electronics Command, Fiber-Optics Network Branch, Fort Monmouth, \\ New Jersey 07703-5000
}

(Received 12 January 1988; accepted for publication 22 March 1988)

\begin{abstract}
By using molecular beam epitaxy and post-growth annealing techniques, we have successfully made GaAs interdigitated photoconductive detectors on D-shaped silica fibers. The detectors exhibit low leakage current and interna! photoconductive gains of up to 15 are measured. To our knowhedge this is the first realization of active III.V optoelectronic devices on fibers and opens up the possibility of realizing optoclectronic integration and circuits directly on fibers.
\end{abstract}

Since optical signal transmission provides high speed and crosstalk-free propagation with very high noise immunity, the use of optical techniques to interconnect electronic integrated circuits and systems for applications in computation and signal processing is receiving increasing attention. Optical systems have many interesting advantages over their electronic counterparts. The inherent paralleiism and nearly limitless bandwidth are attractive for the development of high-speed integrated circuits. In situations where multiple taps from a transmission line are required, optical approaches do not have the capacitive loading effect of electronics. The tremendous progress in fiber optics, optoelectronics, and optoelectronic integrated circuits (OEIC's) suggests that optical fibers may emerge as the most versatile interconnect element. To date, however, optical fibers have mostly been used as passive links for the transmission of optical signals. For many applications, and in particular in OEIC's, it would be of tremendous advantage to design active elements or optoelectronic devices on the transmitting signals. On-board detection, signal processing, and transmission of part of the signals, if possible, would be extremely useful functions for the future developments in optical communication and computing.

One alternate technigue is to route the fibers through active optoelectronic chips, which was recently demonstrated by us.' However, it does nor provide the flexibility obtained by putting the devices on fibers. There have been recent demonstrations of directly sputtered $\mathrm{ZnO}$ piezoelectronic transducers and switchable fber-optic taps on single-mode fibers. ${ }^{2,3}$ We have recently investigated the deposition of MI-V optoelectronic device structures on $\mathrm{SiO}_{x}$ films on Si substrates by molecular beam epitaxy (MBE). The successful operation of detectors made on $\mathrm{SiO}_{x}$ has motivated us to deposil device structures direcly on silica fibers. In this letter we report the molecular beam epitaxial deposition, fabrication, and performance characteristics of interdigitated photoconductive detectors on these fibers.

The optical fibers employed in our experiments were D. shaped monomode silica fibers from Andrew Corporation. This fiber is of special interest because it has a $80 \mu \mathrm{m}$ flat surface and the $2.0 \mu \mathrm{m} \times 1.0 \mu \mathrm{m}$ elliptical core is approximately $10 \mu \mathrm{m}$ underneath the flat surface. Therefore, with the fields normally confined within the guiding core region, the removal of a small amount of material from the surface of the $\mathrm{D}$ will cause the fields to leak to the surface. To prepare the fiber for MBE growth, a short length $(4-6 \mathrm{~cm})$ of it is stripped and cleaved at both ends and is etched in buffered HF (10\%) solution to remove part of the cladding material. The etching time must be carefully controlled so that oniy a
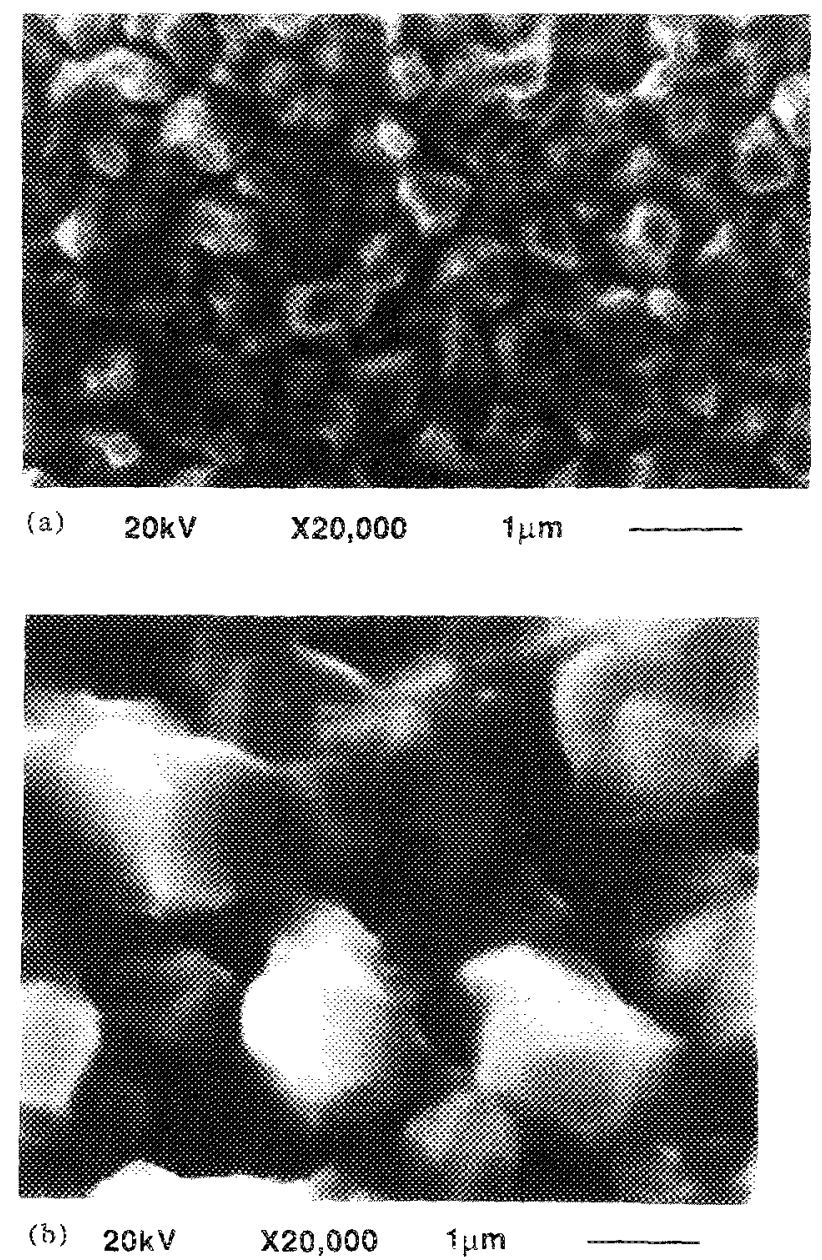

FKG. 1. SEM photomicrographs of GaAs on silica fibers: (a) as grown; (b) lamp annealed at $900^{\circ} \mathrm{C}$ for $10 \mathrm{~s}$ in argon. 
few microns of the cladding layer remain on the fiat surface. For MBE growth of GaAs on it, the fiber is mounted on a Si wafer with the flat surface of the $\mathrm{D}$ facing up and parallel to the surface. This was done with a small amount of highpurity In applied at both ends to hold the fiber against the supporting wafer.

The mounted fiber was introduced in the growth chamber of a Riber $2300 \mathrm{MBE}$ system and growth of GaAs on the fiber was initiated at a very slow rate of $0.1 \mu \mathrm{m} / \mathrm{h}$ at an elevated temperature of $680^{\circ} \mathrm{C}$ to ensure large grain size polycrystal nucleation." After approximately $0.5 \mu \mathrm{m}$ deposition, the growth rate was gradually increased to $0.5 \mu \mathrm{m} / \mathrm{h}$ and another $0.5 \mu \mathrm{m}$ GaAs was deposited. Finally $1.5 \mu \mathrm{m}$ GaAs was grown at $1 \mu \mathrm{m} / \mathrm{h}$ at the nomal temperature of $620^{\circ} \mathrm{C}$. A total thickness of $2.5 \mu \mathrm{m} \mathrm{GaAs}$ was deposited on the flat surface of the fiber. After removal of the sample from the MBE system, the fiber was removed from the Si wafer by heating on a hot plate to melt the In.

Figure 1(a) shows the scanning electron microscope (SEM) photomicrograph of the as-grown GaAs on the fiber. The deposited GaAs is polycrystalline and unsuitable for making devices. To improve the material quality of the GaAs, the sample was annealed. This was achieved by mounting the fiber in a groove in a GaAs wafer and covering it with another GaAs wafer to provide an As overpressure. The sample was annealed at $900^{\circ} \mathrm{C}$ for $10 \mathrm{~s}$ in a halogen lamp annealing station. Figure 1 (b) shows the SEM picture of annealed GaAs on the fiber. It is observed that although the material remains polycrystalline, the grain size is substantially expanded after the rapid thermal amealing.

To fabricate interdigitated GaAs photoconductive detectors on the fiber, the latter was positioned in a $V$-groove made on a Si wafer by preferential etching, ensuring that the fiat surface of the D-fiber was almost in the same level as the supporting wafer. This is important for the subsequent planar technology to make devices. The arrangement is shown schematically in Fig. 2. After positioning the fiber in the groove, a Iit tle photoresist was applied to the ends of the

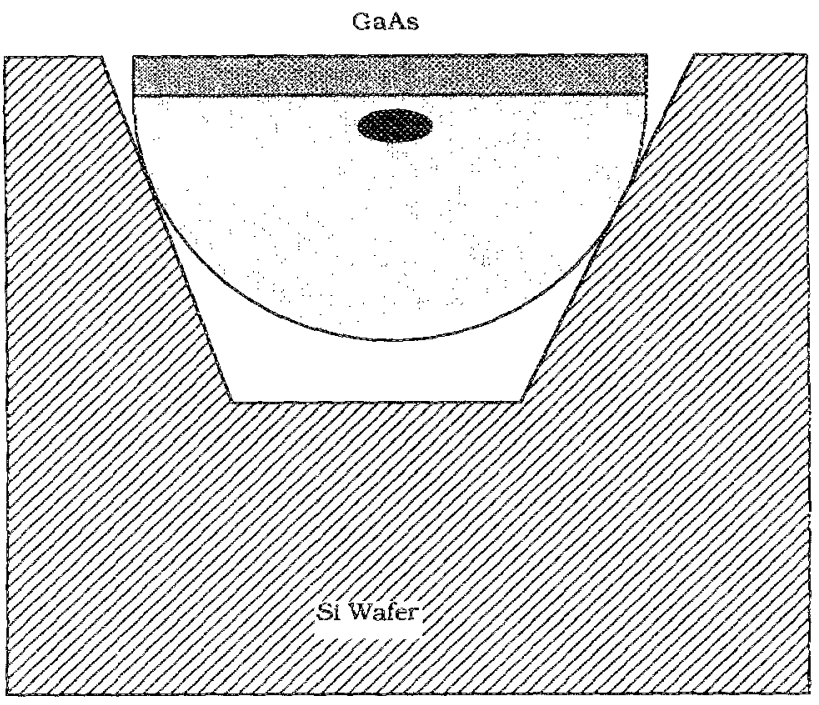

FIG. 2. Schematic of D-fiber with GaAs deposited on it placed in a groove in a Si wafer for lithography.

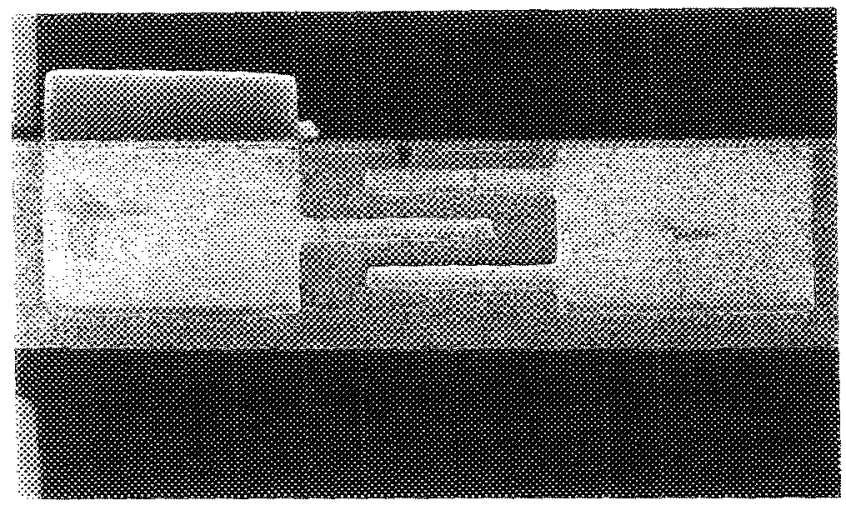

$10 \mathrm{KV} \times 370$

$100 \mu \mathrm{mr}$

FIO. 3. Polycystalline GaAs interdigitated photoconductive detector made on silica fiber by MBE and photolithography. The interelectrode spacing is $15 \mu \mathrm{m}$.

fiber and baked to secure the fiber in the groove. Photoresist was then spun on the whole wafer and standard hithography was used to pattern the detector configuration. After electron beam evaporation of $\mathrm{Ge} / \mathrm{Au} / \mathrm{Ni} / \mathrm{Au}$ contacts, lift-off technology was employed to form the interdigitated electrodes of the photoconductive detectors, as shown in Fig. 3. The interelectrode spacing is $15 \mu \mathrm{m}$. The de characteristics of the detectors were next measured. Without illumination, the leakage current in the devices was around $30 \mu \mathrm{A}$ at a bias of $20 \mathrm{~V}$. The device photocurrent was measured by focusing monochromatic light onto the device surface. It was found that the photocument increased with increasing bias. From the measured photocurrents, and those of a calibrated Si photodetector, the de photoconductive gain of the detector was obtained. Figure 4 depicts the variation of this gain with bias at a wavelength of $0.63 \mu \mathrm{m}$. It is seen that the external photoconductive gain is about 15 at $20 \mathrm{~V}$ bias in the present structure, which is quite acceptable for most apphications. To ensure that the observed photocurrent behavior did not result from the fiber itself, an identical electrode pattern was

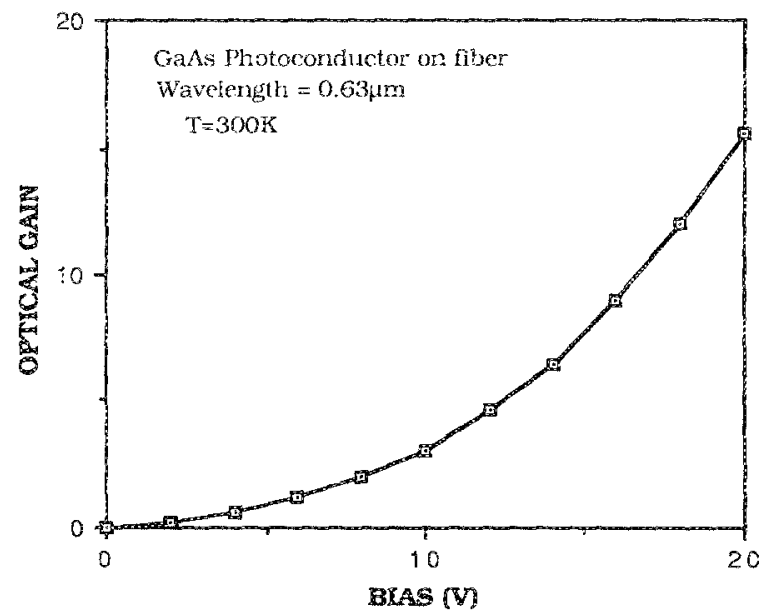

FIG. 4. Dependence of measured optical gain on bias voltage in GaAs phoroconductors on silica fiber. 
fabricated directly on the fibers and tested. No photoconductivity was observed. This confirms the role of the GaAs devices on the fiber. Some comments should be made regarding the observed variation of the photoconductive gain with applied bias. The variation is aimost identical to the variation of the dark current with bias in this device. It is believed that ihis complex behavior results from the transport across grain boundaries in the polycrystalline materials. ${ }^{5}$ At low voltages, carriers may be trapped at the grain boundaries, resulting in a lowering of the gain. The gain increases as these carriers are released from the traps.

It should be remembered that the main role of such detectors will be to detect optical signals transmitted through the fiber. The devices fabricated in the course of this work could not be tested in this mode since light leaking out through the cladding could not generate any photocurrent in the devices with the contacts on the top surface. Ideally, the detector should be placed on the core material in a locally etched recess. Also, the thickness of the layer should be thin enough $(1.0-1.5 \mathrm{~km})$ so that the photogeneratcd carriers can be collected by the contacts on the top surface. We are now in the process of fabricating thinner mesa-etched devices with a modified contact configuration. The other important consideration is that the refractive index of GaAs or other semiconductors used should be higher than the core material of the fiber at the wavelength of operation. With
GaAs or other II-V materials, this will not pose a problem. It is important to realize that although we have not shown here the detection of light guided by the fiber, we demonstrate for the first time that by using MBE and post-growth annealing technique, it is possible to fabricate reliable and useful detectors directly on these fibers. This was possible from the knowledge gained from our initial experiments of MRE deposition on SiO $_{x}$ films on Si substrates.

in conclusion, we have demonstrated that GaAs photodetectors deposited by molecular beam epitaxy on D-shaped silica optical fibers have good perfornance characteristics. It is possible to integrate such devices with electronic components made alongside on the supporting GaAs or $\mathrm{Si}$ wafers, and this work is in progress in our laboratory.

The authors wish to thank Andrew Corporation for supplying the D-shaped fiber samples. This work is being supported by the Army Research Office under contract DAAL03-86-K-0105.

${ }^{1}$ P. K. Bhattacharya, W-QLi, D. A. Weinberger, and D. J. Ellott, Electron. Lett. 22, 1107 (1986).

-3. L. Heffner and B. T. Khuri-Yakub, Appl. Phys. Lett. 48, 1422 (1986). 37. L. Heftner and G. S. Kino, Opt. Lett. 12, 208 (1987).

A. Chin, F. X. Bhattacharya, and G. P. Kothiyal, J. Appl. Phys. 62, 1416 (1987).

${ }^{\circ}$ C. H. Seager and D. S. Ginlcy, Appl. Phys. Lett. 34, 337 (1979); C. H. Seager and G. E. Fike, Appl. Phys. Lett. 35, 709 (1979). 